\title{
Moving towards a synchronized left ventricle
}

\author{
Marat Fudim, MD, ${ }^{a, b}$ and Salvador Borges-Neto, $M^{c}$ \\ a Department of Medicine, Duke University Medical Center, Durham, NC \\ b Duke Clinical Research Institute, Durham, NC \\ c Department of Radiology, Duke University Medical Center, Durham, NC
}

Received Apr 15, 2019; accepted Apr 15, 2019

doi: $10.1007 / \mathrm{s} 12350-019-01749-1$

\section{See related article, pp. 419-430}

Left ventricular mechanical dyssynchrony (LVMD) characterizes the difference in timing of mechanical contraction between different segments of the left ventricle. Notably, LVMD is not equal to electrical dyssynchrony, which is commonly measured by the QRS duration. While both are commonly present at the same time, they can also present independently of each other. ${ }^{1,2}$ Dyssynchronous mechanical systolic contraction is of relevance due to its relationship with adverse outcomes and hypothetical role in patient selection for and procedural guidance of cardiac resynchronization therapy (CRT). Asynchronous mechanical contraction results in an impaired left ventricular contractile efficiency.

Gated Single Photon Emission Computed Tomography (GSPECT) Myocardial Perfusion Imaging (MPI) is used to diagnose and determine prognosis in epicardial coronary artery disease $(\mathrm{CAD}){ }^{3}$ Over the last decade, advances in image processing have allowed an assessment of LVMD via post-processing and creation of series of three-dimensional images of the left ventricle that correspond to sequential time points in a cardiac cycle. The so-called phase analysis determines the variability in the timing of contraction of different LV segments. ${ }^{4}$ Notably, recent advances now also allow the analysis of diastolic LVMD, which identifies asynchronous LV relaxation resulting in impaired LV filling. Importantly, systolic and diastolic LVMD have different

Reprint requests: Marat Fudim, MD, Department of Medicine, Duke University Medical Center, 2301 Erwin Road, Durham, NC, 27710; marat.fudim@dm.duke.edu

J Nucl Cardiol 2020;27:431-3.

$1071-3581 / \$ 34.00$

Copyright (c) 2019 American Society of Nuclear Cardiology. underlying mechanisms and determinants and have independent predictive utility. ${ }^{5-7}$

Measuring LVMD has a prognostic value in a number of patient cohorts. Patients with heart failure and a reduced ejection fraction without history of myocardial infarction, who had significant LVMD had a higher risks of cardiac events irrespective of QRS width and ejection fraction. ${ }^{8}$ In a separate cohort of patients with coronary artery disease, GSPECT-measured LVMD had a relationship with all-cause mortality and cardiovascular mortality independent of electrical dyssynchrony. ${ }^{9}$ Likewise, a number of studies confirmed that LVMD measured by GSPECT MPI in patients with ischemic and non-ischemic cardiomyopathies was an independent predictor of clinical outcomes. ${ }^{10,11}$

An advantage of using GSPECT MPI over other techniques such as echocardiography to measure LVMD is the low inter-observer variability, the ability for postprocessing of images, the ability to identify scar, and quantify ischemia. Taken together, this could provide clinicians some of the key tools to guide optimal CRT lead placement as both contractile reserve and delayed contractility are central factors for appropriate therapeutic response. ${ }^{12,13}$

CRT is most commonly used in the setting of a left bundle branch block (LBBB) in patients with systolic heart failure. LBBB induces electrical and mechanical ventricular dyssynchrony, with detrimental effects in an already failing LV. ${ }^{14}$ The intent of CRT is to improve coordination of global LV contraction ${ }^{15}$ and ideally it is doing so by targeting/stimulating areas of the LV that have the greatest delay in contraction. In clinical practice, the greatest delay of contraction is usually determined without imaging guidance and with support of electrical mapping of some form.

Among patients who meet Class I indications for CRT, $30 \%$ to $40 \%$ of patients fail to show an improvement in clinical symptoms and cardiac function. ${ }^{16}$ This suggest continued efforts to find more efficient ways to select candidates for CRT and 
improved ways to deliver left ventricular leads to the target areas.

The study by Wang et al. presents an elegant exploration of two central questions to current CRT: (A) Does non-invasive GSPECT MPI perform similarly well to invasive electroanatomical mapping (latest electrical activation)? (B) Does a targeted left ventricular lead placement in the location of the greatest mechanical delay provide any hemodynamic benefit over "untargeted" placement in the lateral wall?

In a small sample of pigs, the investigators induced an infarct in the left anterior descending artery (LAD). Following the infarct, the investigators (not surprisingly) found greater degree of LVMD. The first finding was that the locations of greatest mechanical and electrical dyssynchrony correlated very well with each other. Second, the ventricular contractility (dP/dtmax) increased significantly compared to that before CRT when the CRT left ventricular electrode was implanted in viable segments with the greatest degree of LVMD. This was significantly higher than a standardized placement of the left ventricular lead in the viable segments of the lateral wall or scar.

The present work supports several prior analyses and its implications go beyond the good correlation of electrical and mechanical dyssynchrony. As previously suggested, lack of response to CRT, despite meeting traditional criteria for therapy, may in parts be due to the absence of LVMD. ${ }^{17}$ This implies that lead placement during CRT could benefit from LVMD guidance.

Some limitations of the present analysis need to be emphasized: First, the sample size was small. Second, LBBB was induced via an LAD infarct. This does not however represent the reality in most patients undergoing CRT. In humans, LAD disease is commonly accompanied by multi-vessel disease, creating heterogenous scar. Third, in clinical practice, the left ventricular lead is rarely placed in the lateral wall with complete disregard of electrical delay. Thus, the comparator group chosen here does not fully represent clinical practice but serves well in this animal model. Finally, an important consideration is that left ventricular pacing was performed epicardially and not transvenously. It means that the investigators performed pacing through lead placement directly on the heart, without restriction by coronary venous anatomy. In realworld practice, the limitations set by the coronary veins is one of the MAIN limitations to optimizing left ventricular lead placement.

What is needed next? We now need prospective human data to understand whether LVMD-guided lead placement can enhance outcomes. This cannot be done in disregard of human anatomy. Thus, it is even more exciting that there are several efforts underway that attempt to do just that. This includes attempts via (A) intra-procedural integration of coronary venous electroanatomical mapping with delayed enhancement cardiac magnetic resonance imaging, ${ }^{18}$ and (B) fusion of venous anatomy on fluoroscopy venograms with GSPECT MPI LVMD to guide left ventricular lead placement. ${ }^{19}$

\section{Disclosure}

Dr. Fudim has no relevant disclosures and Dr. BorgesNeto is supported by a GE Healthcare grant.

\section{References}

1. Fudim M, Borges-Neto S. A troubled marriage: When electrical and mechanical dyssynchrony don't go along. J Nucl Cardiol 2018.

2. Sillanmaki S, Lipponen JA, Tarvainen MP, Laitinen T, Hedman M, Hedman A, et al. Relationships between electrical and mechanical dyssynchrony in patients with left bundle branch block and healthy controls. J Nucl Cardiol 2018.

3. Beller GA, Zaret BL. Contributions of nuclear cardiology to diagnosis and prognosis of patients with coronary artery disease. Circulation 2000;101:1465-78.

4. Naya M, Manabe O, Koyanagawa K, Tamaki N. The role of nuclear medicine in assessments of cardiac dyssynchrony. J Nucl Cardiol 2018;25:1980-7.

5. Fudim M, Fathallah M, Shaw LK, Liu PR, James O, Samad Z, et al. The prognostic value of diastolic and systolic mechanical left ventricular dyssynchrony among patients with coronary heart disease. JACC Cardiovasc Imaging 2018.

6. Yu CM, Zhang Q, Yip GW, Lee PW, Kum LC, Lam YY, et al. Diastolic and systolic asynchrony in patients with diastolic heart failure: A common but ignored condition. J Am Coll Cardiol 2007;49:97-105.

7. Lee JH. Left ventricular diastolic dyssynchrony in post-myocardial infarction patients: Does it predict future left ventricular remodeling? J Cardiovasc Ultrasound 2016;24:193-4.

8. Bader H, Garrigue S, Lafitte S, Reuter S, Jais P, Haissaguerre M, et al. Intra-left ventricular electromechanical asynchrony. A new independent predictor of severe cardiac events in heart failure patients. J Am Coll Cardiol 2004;43:248-56.

9. Hess PL, Shaw LK, Fudim M, Iskandrian AE, Borges-Neto S. The prognostic value of mechanical left ventricular dyssynchrony defined by phase analysis from gated single-photon emission computed tomography myocardial perfusion imaging among patients with coronary heart disease. J Nucl Cardiol 2017;24:48290.

10. AlJaroudi W, Alraies MC, Menon V, Brunken RC, Cerqueira MD, Jaber WA. Predictors and incremental prognostic value of left ventricular mechanical dyssynchrony response during stress-gated positron emission tomography in patients with ischemic cardiomyopathy. J Nucl Cardiol 2012;19:958-69.

11. Goldberg AS, Alraies MC, Cerqueira MD, Jaber WA, Aljaroudi WA. Prognostic value of left ventricular mechanical dyssynchrony by phase analysis in patients with non-ischemic cardiomyopathy with ejection fraction $35-50 \%$ and QRS $<150 \mathrm{~ms}$. J Nucl Cardiol 2014;21:57-66.

12. Bleeker GB, Kaandorp TA, Lamb HJ, Boersma E, Steendijk P, de Roos A, et al. Effect of posterolateral scar tissue on clinical and 
echocardiographic improvement after cardiac resynchronization therapy. Circulation 2006;113:969-76.

13. Murphy RT, Sigurdsson G, Mulamalla S, Agler D, Popovic ZB, Starling RC, et al. Tissue synchronization imaging and optimal left ventricular pacing site in cardiac resynchronization therapy. Am J Cardiol 2006;97:1615-21.

14. Yu CM, Hayes DL. Cardiac resynchronization therapy: State of the art 2013. Eur Heart J 2013;34:1396-403.

15. Sade LE, Demir O, Atar I, Muderrisoglu H, Ozin B. Effect of mechanical dyssynchrony and cardiac resynchronization therapy on left ventricular rotational mechanics. Am J Cardiol 2008;101:1163-9.

16. Bertini M, Hoke U, van Bommel RJ, Ng AC, Shanks M, Nucifora $\mathrm{G}$, et al. Impact of clinical and echocardiographic response to cardiac resynchronization therapy on long-term survival. Eur Heart J Cardiovasc Imaging 2013;14:774-81.

17. Emkanjoo Z, Esmaeilzadeh M, Mohammad Hadi N, Alizadeh A, Tayyebi M, Sadr-Ameli MA. Frequency of inter- and intraventricular dyssynchrony in patients with heart failure according to QRS width. Europace 2007;9:1171-6.

18. Nguyen UC, Mafi-Rad M, Aben JP, Smulders MW, Engels EB, van Stipdonk AM, et al. A novel approach for left ventricular lead placement in cardiac resynchronization therapy: Intraprocedural integration of coronary venous electroanatomic mapping with delayed enhancement cardiac magnetic resonance imaging. Heart Rhythm 2017;14:110-9.

19. Zhou W, Hou X, Piccinelli M, Tang X, Tang L, Cao K, et al. 3D fusion of LV venous anatomy on fluoroscopy venograms with epicardial surface on SPECT myocardial perfusion images for guiding CRT LV lead placement. JACC Cardiovasc Imaging 2014;7:1239-48.

Publisher's Note Springer Nature remains neutral with regard to jurisdictional claims in published maps and institutional affiliations. 